\title{
L'intégration des IUFM aux universités illustrée par un exemple
}

Pierre Fontes (pierre.fontes@wanadoo.fr)

Professeur honoraire (université Paris-Sud et IUFM de l'académie de Versailles)

Élu Sgen-CFDT au CA de l'IUFMV et à sa commission permanente de 1995 à 2007

La loi d'orientation

et de programmation

sur l'avenir de l'école

promulguée en avril 2005

prévoit que, dans

un délai de trois ans,

chacun des trente et un

instituts universitaires

de formation des maîtres

(IUFM) sera intégré

à une université de

l'académie où se situe

son siège. Établissement

public à caractère

administratif créé

par la loi d'orientation

sur l'éducation de juillet 1989, un IUFM devient

école interne de son

université d'intégration.

L'histoire et les enjeux

de cette intégration

sont présentés

et illustrés par l'exemple de l'IUFM de l'académie

de Versailles,

l'un des deux premiers

à être intégré.

\section{9 : création des IUFM, rattachés à des universités}

Les instituts universitaires de formation des maitres (IUFM), créés par la loi d'orientation sur l'éducation de juillet 1989 sont, pour la plupart, mis en place à la rentrée 1991. Dans chacune des académies, vingt-neuf à l'époque, est mis en place un IUFM auquel sont dévolus les moyens matériels et humains des anciennes écoles normales d'instituteurs et des écoles normales nationales d'apprentissage. Les préparations aux concours de professeurs des écoles (réservés aux titulaires d'une licence et remplaçant à cette même date les concours de recrutement des instituteurs) et de professeurs de lycées professionnels sont assurées sur des sites de l'IUFM. Les préparations aux différents certificats d'aptitude à l'enseignement secondaire (CAPES) sont, pour la plupart, maintenues dans des universités. Celles des agrégations relèvent toujours des écoles normales supérieures et/ou des universités. Les lauréats de tous les concours de recrutement, agrégés inclus, deviennent professeurs stagiaires en deuxième année d'IUFM à l'issue de laquelle ils sont, en général, titularisés.

Chaque IUFM est rattaché à toutes les universités de l'académie par des conventions qui régissent les relations entre ces établissements d'enseignement supérieur dans le domaine de la formation des maîtres : préparations aux concours de recrutement, échanges de service d'enseignants-chercheurs ou d'enseignants, collaborations dans les activités de recherche.

Établissement public à caractère administratif, placé sous la responsabilité d'un directeur nommé par le ministre en charge de l'éducation, doté d'un conseil d'administration (CA), présidé ès qualités par le recteur de l'académie, et d'un conseil scientifique et pédagogique, un IUFM n'est donc pas, alors, un institut au sens de la loi sur l'enseignement supérieur de 1984, comme le sont, par exemple, les instituts universitaires de technologie (IUT).

Dès leur création, les IUFM sont la cible de nombreuses critiques, parfois contradictoires : des membres des corps d'inspection se considérant comme dépossédés d'une partie de leur contrôle sur la formation des enseignants ; des universitaires restant attachés à la seule dimension disciplinaire de leur métier ; des campagnes de presse dénonçant tour à tour soit la nature de la formation, qualifiée d'inadaptée, soit une coupure entre cette formation, trop disciplinaire, et le travail quotidien des enseignants, soit un " pédagogisme " considéré comme l'idéologie dominante parmi les enseignants d'IUFM.

À plusieurs reprises sont élaborés des projets de modifications profondes des IUFM, voire la demande de leur disparition, au gré des alternances dans la vie politique du pays. En 2003, sous le ministère Ferry, cette convergence d'attaques a bien failli obtenir satisfaction, avec un projet d' « écoles professionnelles » qui auraient redonné l'essentiel du contrôle de la formation des maitres aux recteurs et aux corps d'inspection, en réduisant la préparation au métier à une espèce de compagnonnage entre des enseignants supposés experts et les débutants.

Ainsi s'accumulent des entraves à l'évolution nécessaire du dispositif de formation des maitres. Les enseignants-chercheurs affectés dans les IUFM représentent une minorité du corps enseignant (de l'ordre de 10 à $15 \%$ ) et le caractère universitaire de ces établissements reste faiblement assuré.

\section{5 : du rattachement à l'intégration}

La loi d'orientation et de programmation sur l'avenir de l'école stipule que, dans un délai de trois ans à compter de sa promulgation en avril 2005, les IUFM, maintenant au nombre de trente et un, doivent être intégrés dans une des universités de leur académie.

Ce processus est aujourd'hui en voie d'achèvement. En mai 2008, à six exceptions près ${ }^{(1)}$, les décrets d'intégration sont publiés. L'intégration apparaît comme une nouvelle étape dans l'universitarisation de la formation des maitres, et permet une véritable formation qui ne se réduit pas à un simple apprentissage sur le tas. À l'heure où se développe le système 
licence, master, doctorat, cette intégration universitaire, souhaitée à des degrés divers par une partie des personnels enseignants, est particulièrement bienvenue. Elle donne la perspective d'accéder à une validation de cursus autre que la seule certification d'enseignant : aux étudiants qui échoueront une ou plusieurs fois aux concours, ainsi qu'aux enseignants qui, après quelques années d'exercice, souhaiteront changer d'activité. Dans ce métier, comme dans tous les autres, la diversification des sorties possibles peut certainement constituer un appel bénéfique au niveau de l'entrée.

À l'instar des IUT, mais avec des effectifs qui peuvent atteindre plusieurs milliers d'usagers, étudiants et professeurs stagiaires, les IUFM deviennent, alors, une école interne de leur université d'intégration. D'établissement autonome, disposant d'un directeur, d'un secrétaire général, d'un agent comptable, d'un budget soumis à l'approbation de son seul CA, ils deviennent composante d'une université.

Malheureusement, sous le ministère Robien, des divergences entre la Direction générale à l'enseignement supérieur (Dges) favorable à l'intégration, et la Direction générale à l'enseignement scolaire (Dgesco), qui n'a pas encore renoncé à l'idée des écoles professionnelles, conduisent à une longue période d'hésitation, voire de temporisation, également entretenue par la conférence des directeurs d'IUFM soucieuse de conserver le pouvoir acquis. À la fin de l'année universitaire 2005-2006, le processus d'intégration est au point mort. C'est alors que les recteurs reçoivent pour consigne de l'accélérer.

Avant d'arriver à une intégration effective, de nombreuses questions sont à régler qui vont du choix de l'université d'intégration à la mise en place de nouvelles structures comme le conseil d'école. Pour certains, l'intégration représente une réduction d'autonomie ou une perte de pouvoir ou une suppression de postes. Pour les universités, à l'époque où les effectifs n'augmentent plus, la perspective de l'inscription de centaines ou même de milliers d'étudiants supplémentaires, assortie $\mathrm{du}$ transfert des emplois d'enseignants, enseignants-chercheurs et administratifs de l'IUFM, apparaît évidemment plus comme une aubaine que comme une charge supplémentaire. "Attendez-vous à être courtisé ! " déclare un recteur lors d'un CA de l'IUFM qu'il préside.
Les modalités de cette intégration sont très diverses, selon les IUFM, en fonction des acteurs locaux. Si l'objectif est parfaitement souhaitable, sa mise en œuvre peut se révéler chaotique, comme l'illustre l'exemple ci-dessous.

\section{L'IUFM de l'académie de Versailles intégré à l'université de Cergy-Pontoise}

Les IUFM des académies d'Aix-Marseille et deVersailles sont les premiers à voir publier leur arrêté d'intégration au $1^{\mathrm{er}}$ janvier 2007.

L'académie de Versailles représente environ $10 \%$ de l'ensemble du système éducatif français et l'IUFM de l'académie de Versailles (IUFMV) est le plus important du pays ${ }^{(2)}$. En nombres arrondis, il comprend 7000 étudiants et stagiaires, 300 personnels non enseignants et 400 enseignants à temps plein, dont 50 maîtres de conférence et 10 professeurs des universités.

Cette académie comporte cinq universités, deux anciennes, celles de Nanterre, essentiellement littéraire, et de Paris-Sud, surtout scientifique ; trois " nouvelles universités » de caractère pluridisciplinaire plus ou moins marqué : Cergy-Pontoise, Évryval d'Essonne, Versailles-Saint-Quentinen-Yvelines.

La concertation entre les présidents de ces universités a maintenu l'incertitude, excepté en ce qui concerne celle d'Évry-val d'Essonne qui n'a jamais été candidate pour intégrer l'IUFMV. Les candidatures des deux anciennes se sont annihilées, aucune ne souhaitant que l'autre l'emporte. De plus, la candidature de Paris-Sud a été mollement défendue, par suite d'un contresens sur les conséquences et les difficultés de l'intégration.

À la fin de l'année universitaire 20052006, rien n'est clairement défini. Le processus d'intégration s'emballe alors brutalement. Les personnels de l'IUFMV, directeur compris, apprennent au cours de l'été 2006 que l'intégration se fera à l'université de Cergy-Pontoise. En septembre 2006, le CA de l'IUFMV en est informé. Lui reste à entériner cette décision, préalablement élaborée par le recteur de l'académie de Versailles avec le président de l'université de Cergy-Pontoise dont l'IUFMV intégré va constituer environ $40 \%$ des effectifs. C'est un rapport de forces a priori très favorable à l'IUFMV. Mais si l'intégration reste éminemment souhaitable, les conditions de sa réalisation sont particulièrement exécrables. Les personnels de l'IUFMV, à tort ou à raison, se sentent un peu abandonnés, voire même trahis. Les relations entre les services s'établissent dans un mélange subtil de franche collaboration et de réticence affichée. La commission permanente de l'IUFMV, élargie au recteur et aux organes de direction du rectorat, est chargée d'étudier les problèmes à résoudre et d'élaborer les statuts de l'école interne. Elle s'empêtre dans des débats confus et des combats d'arrière-garde menés par des représentants syndicaux opposés de fait à l'intégration. Le travail qui aurait dû être plus efficace et conduit dans l'enthousiasme se révèle long, difficile et parfois même stérile. Par exemple, les enjeux majeurs de la " mastérisation " de la formation ne sont évoqués qu'au passage.

Mi-mai 2008, le conseil d'école est en place et son président est élu. Mais six semaines après un vote très serré pour l'élection du directeur de l'IUFMV, sa nomination n'est toujours pas confirmée par le ministère. L'ancien directeur, devenu administrateur provisoire depuis janvier 2007, fête son départ à la retraite.

Parallèlement, à Aix-Marseille, la volonté de réussir l'intégration est présente à l'université comme à l'IUFM. Désirée de part et d'autre et bien préparée, elle se réalise dans des conditions à la fois plus dynamiques et plus paisibles.

(1) Elles concernent la fusion d'universités pour l'IUFM d'Alsace et des situations exotiques (Antilles, Corse, Guyane et Pacifique).

(2) Il comporte cinq sites, un à Saint-Germain-en-Laye (Yvelines), un à Étiolles (Essonne), deux à Antony (Hauts-de-Seine) et un à Cergy (Val-d'Oise).

\section{Quelques références}

Les lois citées

Loi $n^{\circ}$ 84-52 du 26 janvier 1984,

dite Loi Savary

Loi $n^{\circ}$ 89-486 du 10 juillet 1989 ,

dite Loi Jospin

Loi n² 2005-380 du 23 avril 2005,

dite Loi Fillon

Le portail des IUFM

http://www.iufm.fr/

Un article

Bulletin SFP 152 (2005-2006), pp. 26-27. 